\title{
Oligodendrogliomas with Abundant Refractile Eosinophilic Granular Cells
}

\author{
Kelvin Au, Juan Bilbao, Todd Mainprize, Michael Schwartz, Julia Keith
}

Can J Neurol Sci. 2013; 40: 746-748

Refractile eosinophilic granular cells, when present in abundance in a glioma, may trigger a diagnosis of the relatively familiar and inherently malignant 'granular cell astrocytoma'. The following two cases remind us that refractile eosinophilic granular cells are not unique to granular cell astrocytoma but can also dominate the histology of oligodendroglioma confirmed by $1 \mathrm{p} 19 \mathrm{q}$ loss, and the immunohistochemical and ultrastructural A features of two such cases are reviewed. Refractile eosinophilic granular cells are not to be confused with the similar sounding term of eosinophilic granular bodies, the hyaline PAS positive a c c u m u l a t i on s frequenting low grade glial and glioneuronal tumours.
A 62-year-old woman had a sub-total resection of a ring enhancing right temporal brain tumour (Figure 1a). Histologic sections showed an infiltrative cellular tumour with round yet atypical nuclei, numerous refractile eosinophilic granular cells (rEGC) which dominated the smear preparations (Figure 1b), scattered minigemistocytes and mitoses but lacking necrosis and endothelial proliferation. The immunohistochemical and special staining features of the rEGCs are described in the Table. Electron microscopy showed the rEGC to have a cytoplasm rich in filamentous whorls including osmiophilic structures with the appearance of mini-Rosenthal fibers (Figures 1c, 1d). Polymerase chain reaction (PCR) testing for $1 \mathrm{p} 19 \mathrm{q} \mathrm{LOH}$ showed allelic loss of $1 \mathrm{p}$ (6 of 6) and partial allelic loss of chromosome 19q (1 of 5). The final diagnosis was anaplastic oligodendroglioma, World Health Organization (WHO) grade III.

A 35-year-old man had an excisional biopsy of a $4 \mathrm{~cm}$ focally enhancing lesion of the left deep grey structures (Figure 2a) and sections showed a densely cellular proliferative glioma with a focal myxoid background lacking a classic oligodendroglial morphology. There were numerous intermixed cells with eccentrically located nuclei and ample eosinophilic cytoplasm with coarse refractile granules (Figure 2b). The
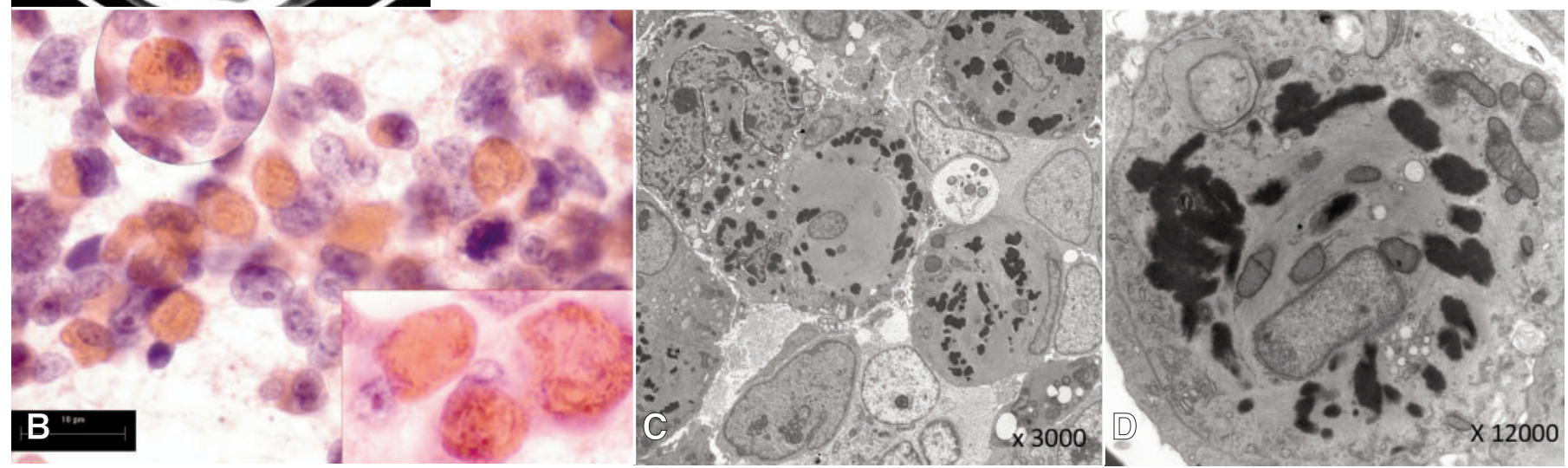

Figure 1: a - axial T1 contrast enhanced MRI showing ring enhancing right temporal brain tumour; $b$ - smears prepared intra-operatively showing lesional glial cells with eosinophilic refractile cytoplasmic inclusions; $c, d$-ultrastructural examination (X3000 and X12000 magnification) showed the refractile eosinophilic cells to contain cytoplasmic filamentous whorls and osmiophilic structures with the appearance of mini-Rosenthal fibers.

From the Department of Anatomical Pathology (KA, JB, JK), Division of Neurosurgery, Department of Surgery (TM, MS), Sunnybrook Health Sciences Centre, and University of Toronto, Toronto, Ontario, Canada

Received November 20, 2012. Final Revisions Submitted April 24, 2013.

Correspondence to: Julia Keith, Room E403, Department of Pathology, 2075 Bayview Avenue, Toronto, Ontario, M4N 3M5, Canada. Email: julia.keith@ sunnybrook.ca. 
Table: Immunohistochemical and special staining qualities of refractile eosinophilic granular cells

\begin{tabular}{l|l|l}
\hline & positive staining & negative staining \\
\hline Case 1 & $\begin{array}{l}\text { GFAP, S100, CD56, synaptophysin, alpha-beta- } \\
\text { crystallin, CD117, panCK, p53 (patchy), vimentin }\end{array}$ & $\begin{array}{l}\text { desmin, actin, EMA, CD68, neurofilament, myosin, NeuN, chromogranin, } \\
\text { PAS and mIDH1(IDH1R132H) }\end{array}$ \\
\hline Case 2 & $\begin{array}{l}\text { GFAP, S100, CD56, synaptophysin, alpha-beta } \\
\text { crystallin, panCK, p53 (patchy), desmin, vimentin a } \\
\text { mIDH1 (IDH1R132H) }\end{array}$ & $\begin{array}{l}\text { actin, EMA, CD68, neurofilament, myosin, NeuN, chromogranin, CD117 } \\
\text { and PAS }\end{array}$ \\
\hline
\end{tabular}

immunohistochemical and special staining features of the refractile eosinophilic cells are described in the Table and shown in Figures 2c-e. Electron microscopy was not undertaken. By PCR the tumour had allelic loss of 1p (4 of 4) and 19q (5 of 5). The final diagnosis was an anaplastic oligodendroglioma, WHO grade III.

Although rEGC have been previously noted and described as an 'astrocyte-like cell' in oligodendroglioma ${ }^{1-3}$, their abundant glial fibrillary acidic protein (GFAP) positive cytoplasm may prompt pathologists to erroneously interpret a lesion as an astrocytoma, potentially resulting in inappropriate prognostic and predictive information being given to a patient and chemotherapy withheld. This risk is especially prudent considering pathologists' collective familiarity with granular cell astrocytoma ${ }^{4}$, and in cases lacking other classic morphologic features of oligodendroglioma. Eosinophilic granular inclusions have also been reported in ependymoma ${ }^{5}$, medulloblastoma ${ }^{6}$, and schwannoma ${ }^{7}$. Yoshida et $\mathrm{al}^{2}$ re-examined 102 gliomas and found some rEGC in $9 \%$ of grade II oligodendrogliomas, $11 \%$ of grade II oligo-astrocytomas, $82 \%$ of grade III oligodendrogliomas and $76 \%$ of grade III oligo-astrocytomas. When present the rEGC were more numerous in the grade III tumours ${ }^{2}$. Testing for $1 \mathrm{p} 19 \mathrm{q} \mathrm{LOH}$ and $\mathrm{mIDH} 1$ was not described in their series. The cytologic features of the rEGC in our two cases are comparable to those described by Yoshida et al. Our ultrastructural findings of cytoplasmic filamentous whorls were analogous to the ellipsoidal pattern of "Rosenthal-like fibres" seen in the Yoshida series ${ }^{2}$ and subsequently described ${ }^{3}$, and differed from both the autophagic vacuoles causing nonrefractile cytoplasmic granules in oligodendroglioma described
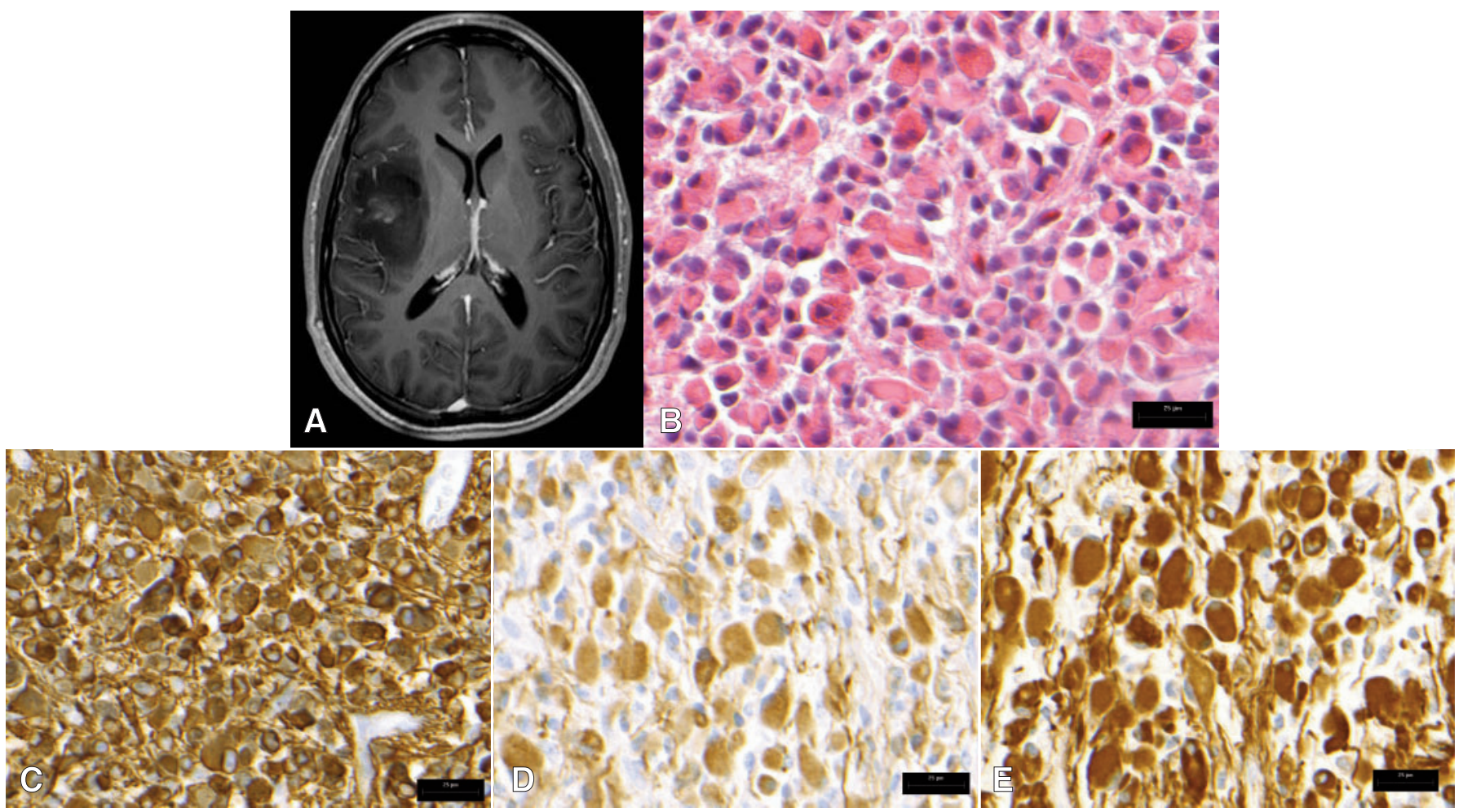

Figure 2: a - axial T1 weighted contrast enhanced MRI showing enhancing brain tumour affecting the left deep grey structures; $b$ - hematoxylin and eosin stained slide showing a glioma with scattered cells with eccentrically located nuclei and ample eosinophilic cytoplasm with coarse refractile granules (arrow); the refractile eosinophilic cells are immunopositive for GFAP (c), panCK (d) and desmin (e). 
by Takei $^{8}$ and the lysosomal granules of granular cell astrocytoma ${ }^{4}$. The previously documented immunohistochemical features of rEGCs include strong GFAP, S100, vimentin and alpha-beta-crystallin expression ${ }^{2}$, comparable to a Rosenthal fibre. Refractile eosinophilic granular cells and the cells of granular cell astrocytoma both express GFAP and S100, but Periodic-acid Schiff (PAS) and CD68 positivity is more supportive of a granular cell astrocytoma diagnosis ${ }^{4}$, and either1p19q LOH and/or electron microscopy may be helpful in resolving this differential diagnosis. We expand the immunohistochemical profile of rEGCs in oligodendroglioma to include synaptophysin, CD56, pan-CK and desmin positivity, and demonstrate their presence in gliomas with variable mIDH1 status and oligodendroglial lineage confirmed by $1 \mathrm{p} 19 \mathrm{q}$ loss. Although these cells could represent neoplastic granular astrocytic cells in a mixed oligoastrocytoma housing 1p19q loss, this report reminds pathologists not to assume that rEGC are reflective of an exclusively astrocytic lineage. Some questions remain, including the pathogenesis of these rEGC cells and whether their presence heralds an altered biologic potential of an oligodendroglioma.

\section{REFERENCES}

1. Kojima H, Mori K, Fukudome N, Iseki M, Shimizu S. Cytologic characteristics of intracytoplasmic refractile eosinophilic granular bodies in anaplastic oligodendroglioma: a case report. Acta Cytol. 2008;52(4):467-70.

2. Yoshida T, Nakazato Y. Characterization of refractile eosinophilic granular cells in oligodendroglial tumors. Acta Neuropathol. 2001;102(1):11-19.

3. Burger PC, Scheithauer BW, Vogel FS. Tumours. In: Burger PC, Scheithauer BW, Vogel FS, editors. Surgical pathology of the nervous system and its coverings, 4th edition. Philadelphia: Churchill Livingstone; 2002. p. 230-1.

4. Shi Y, Morgenstern N. Granular cell astrocytoma. Arch Pathol Lab Med. 2008;132(12): 1946-50.

5. Shintaku M, Yoshida M, Ikarashi T, Arakawa Y, Nagashima T. Ependymoma with "granular cell" features: Report of two cases. Neuropathology. 2012;32(6):654-1.

6. Rodriguez FJ, Scheithauer BW. Anaplastic medulloblastoma with granular cell change. Acta Neuropathol. 2007;113(1):95-9.

7. Yokoo $\mathrm{H}$, Arai $\mathrm{H}$, Isoda $\mathrm{K}$, et al. Characterization of eosinophilic hyaline droplets in schwannoma. Acta Neuropathol. 2003;105: $170-6$.

8. Takei Y, Mirra SS, Miles ML. Eosinophilic granular cells in oligodendrogliomas. An ultrastructural study. Cancer. 1976;38 (5):1968-76. 